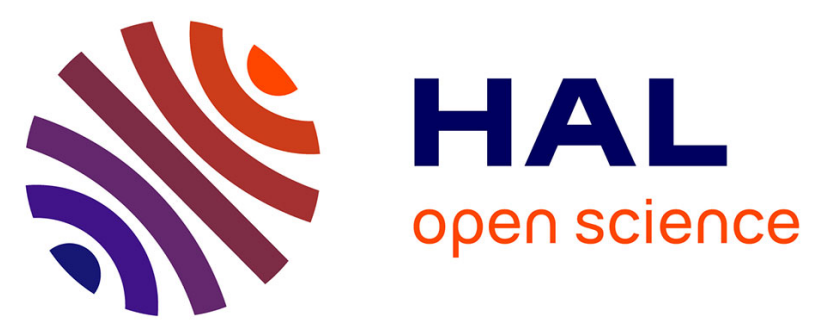

\title{
A Non Destructive Reflectometry Based Method for the Location and Characterization of Incipient Faults in Complex Unknown Wire Networks
}

\author{
Moussa Kafal, Fatme Mustapha, Wafa Ben Hassen, Jaume Benoit
}

\section{- To cite this version:}

Moussa Kafal, Fatme Mustapha, Wafa Ben Hassen, Jaume Benoit. A Non Destructive Reflectometry Based Method for the Location and Characterization of Incipient Faults in Complex Unknown Wire Networks. 2018 IEEE AUTOTESTCON, Sep 2018, National Harbor, United States. pp.8532500, 10.1109/AUTEST.2018.8532500 . cea-03122224

HAL Id: cea-03122224

https://hal-cea.archives-ouvertes.fr/cea-03122224

Submitted on 26 Jan 2021

HAL is a multi-disciplinary open access archive for the deposit and dissemination of scientific research documents, whether they are published or not. The documents may come from teaching and research institutions in France or abroad, or from public or private research centers.
L'archive ouverte pluridisciplinaire HAL, est destinée au dépôt et à la diffusion de documents scientifiques de niveau recherche, publiés ou non, émanant des établissements d'enseignement et de recherche français ou étrangers, des laboratoires publics ou privés. 


\title{
A Non Destructive Reflectometry Based Method for the Location and Characterization of Incipient Faults in Complex Unknown Wire Networks
}

\author{
Moussa KAFAL ${ }^{*}$, Member, IEEE, Fatme MUSTAPHA*, Wafa BEN HASSEN*, and Jaume BENOIT* \\ ${ }^{*}$ CEA, LIST, Laboratoire de Fiabilité et Intégration Capteurs, 91191 Gif-sur-Yvette, France. \\ moussa.kafal@cea.fr
}

\begin{abstract}
During the last decade, vast efforts have been invested in research and industry to detect soft noncritical faults in wiring networks. Although time domain reflectometry based methods (TDR) have been the center stage of such techniques, the capability of characterizing the located faults was still out of reach. In fact, this is so important as it can potentially enable preventive maintenance well before the fault's deterioration to critical dangerous stages. An assessment of the fault's situation becomes possible thus maximizing the system functionality and safety while minimizing the out-of-service time. In this paper, we will propose an approach based on the tenets of TDR and post-processing techniques, namely baselining and optimization based algorithms, to detect, locate and characterize soft faults embedded in complex networks. More importantly, this will be accomplished using a single testing port of a totally unknown network whose extremities are kept connected to their loads. Numerical as well as practical experimental results will be employed to validate the efficiency of the proposed approach.
\end{abstract}

Index Terms-Complex wire networks; fault characterization; Genetic algorithms; Inverse problems; Soft faults.

\section{INTRODUCTION}

Recently, the world is witnessing the fourth Industrial Revolution built on the huge digital uprising which is combining multiple technologies that are leading to unprecedented paradigm shifts in the economy, business, society, and for individuals. In fact, it is involving the transformation of entire systems. Significantly, the electricity landscape is a prime example of the Fourth Industrial Revolution as it is undergoing massive transformations, becoming more complex than ever before, with rapidly evolving technologies. Meanwhile, a reliable, safe, economically competitive and environmentally sustainable electrical power grid is the cornerstone of a modern society.

Regardless the application nature, whether belonging to the transportation sector, robots, industry, power-plants, etc., systems are hosting cumulated lengths of wiring networks responsible for communication, distribution and control operations between all components within a system. For instance, 10 kilometers of cables accommodate in a modern truck, $40 \mathrm{~km}$ in a helicopter and more than $500 \mathrm{~km}$ in a modern civil transport aircraft as the A380 [1], [2]. Although, lots of safety measures have been taken to protect cables from damage, electrical wires are still highly likely exposed to compelling conditions (heating, humidity, etc.) leading to the appearance of anomalies. Such faults may lead to a total interruption of transmission as in the case of open or short circuits (hard faults). Meanwhile soft or incipient faults are the second major family of wiring defects that are characterized by slightly modifying the cable properties without causing a significant impact on the whole system operation as chafes, arcs, etc. They are considered significantly more difficult to detect and may easily evolve into hard faults [3]. Hence, developing robust procedures for the detection of soft faults has become critically important, as an early warning approach for ensuring the safe and reliable operation of electrical systems.

Soft faults have been extensively handled in current literature where reflectometry-based methods are still the cutting edge of most embedded and distributed wire diagnosis systems [4], [5]. Intuitively relying on the analysis of the electrical waves echoed on impedance mismatches (IDis) along a network under test (NUT), Time-Domain Reflectometry (TDR) has a long history with cable diagnosis but require great analysis expertise [6], [7]. In fact, the problem of soft faults is critical and requires in-depth studies since their corresponding reflected weak echoes might be easily masked from reflectometry detection by pseudo echoes generated by higher reflectivity impedance discontinuities like junctions or splitters, etc..

However, there have been a vast development of signal and post-processing tools that can be integrated with TDR methods and have consequently facilitated the process of extracting weak soft faults' signatures. For instance, methodologies not limited to the time-frequency cross-correlation function using the Wigner-Ville transform (WVT) [8], [9], the Self-Adaptive Correlation Method (SACM) [10], the Signature Magnification by Selective Windowing (SMSW) [11] in addition to the Time Reversal (TR) techniques have efficiently succeeded in this mission [12]-[16].

In fact, this can be assumed valid as long as the topology of the NUT is known and easy access to all its extremities can be afforded. Regrettably, enormous wiring network datasheets are lost in lots of applications, while access to more than one port is impossible in some embedded networks as those in nuclear power-plants or airplanes. Although, fair estimates of the fault's positions were still achieved with acceptable location errors, unveiling the nature of the detected soft faults (resistive, inductive, etc.) and estimating their local variation was still unreachable.

Particularly, these are indeed important information as they can help in estimating an approximate useful operational time of the defected cable and accordingly allow an assessment of the cost of waiting versus direct repair. Besides, knowing the nature of a soft fault would allow collecting helpful data that can enable building a clear image of the degradation procedure with time and under different external and internal conditions (heating, humidity, pressure, etc.). Therefore, in order to cope 
with all these limitations, we are going to propose in this paper a technique capable of blindly diagnosing a black-boxed faulty fully loaded NUT, i.e. with loads connected to it's extremities; more importantly using a single testing port.

The method will be composed of two phases. In the first, a TDR measurement of the unknown faulty NUT will be treated in a post-processing algorithm based on the tenets of TDR in conjunction with the Graph theory and the genetic based optimization algorithm (GA) in a TDR-GA methodology. Since the soft fault has a minor contribution on the overall TDR response, we will be able to reconstruct the topology of a healthy version of the tested NUT, with the branch lengths and load impedances. The second phase starts by applying a baselining approach that is taking the difference between the measured faulty TDR response and that of a simulated response computed from the reconstructed healthy NUT. The result will be a reflectometry response amplifying the signature of the soft fault, which once treated with a similar TDR-GA algorithm will enable not only precisely locating the soft fault, but also determining its location, nature and characteristics. The proposed method will be validated by experimental results conducted on single junction network configuration.

\section{PRoblems AND Challenges}

In what follows we will try to brief the main obstacles reflectometry based methods encounter in soft fault diagnosis to converge finally to the solution method we will propose.

\section{A. Network complexity}

It has been demonstrated that the detection of incipient faults is intractable even when dealing with simple networks (single branch cables) due to the fact that the echo amplitude caused by these faults can be in some cases analogous to the noise level or other residual IDis caused artifacts [5], [17]. The situation is expected to worsen once complex networks are addressed which are often found in embedded systems (e.g., vehicles, airplanes, etc.). These usually comprise NUTs containing at least one junction between different branches and are expected to respond to a test signal by a long series of reflected echoes. Unfortunately, these IDis (junctions, loads, etc.) reflected echoes are likely to have bigger order of magnitudes compared to the echoes reflected by weak reflectivity soft faults. Specifically, an increased complexity of the NUT is accompanied by more complex reflectometry responses leading to difficulty in interpretation and poverty in extraction of the soft faults' signatures and thus generating false alarms.

\section{B. Network's structure}

Real life systems usually host wiring networks having specific configurations in the form of a bus, mesh, or tree, etc.. Accordingly, complicated reflectometry responses, characterized by the presence of multiple reflections caused by junctions and other impedance mismatches, are obtained. However, the extraction procedure of weak soft fault signatures became possible, thanks to different post-processing algorithms recently developed and integrated with reflectometry techniques as those listed in the sec. I. On the other hand, a proper application of such techniques rely on the prior knowledge of the network's topology. Unfortunately, this is often unattainable due to sudden updates in wiring installations decisions taken by technicians for some reasons as in the case of buildings or lost wiring maps when dealing with old infrastructures, etc.. Indeed, testing networks of unknown or partially unknown configurations in an effort to detect and locate soft faults is seemingly impossible. Accordingly, after investigating the aforementioned obstacles, how would it be possible to characterize incipient defects embedded in such NUTs?

It is worthy to note that the situation is expected to deteriorate due to testing restrictions imposed by a vast majority of fault diagnosis methods. In fact, this includes disconnecting the NUT's extremities from its loads, forcing specific extremity connections (open or short circuits), accomplishing measurements based on several testing ports and requiring the presence of a healthy prototype of the network, etc. [1], [4], [6]-[11], [15], [16], [18], [19]. Significantly, the authors in [20]-[22] succeeded in proposing a method based on the tenets of TDR, graph theory and optimization based algorithms in order to blindly reconstruct the topology of a wiring network, return precise estimates of branch lengths and retrieve load impedances using a single testing port and without having to disconnect the network from neither its infrastructure nor extremities. The interested reader could return to these paper for a detailed overview. In fact, the technique proposed in [22] will form the first phase of our proposed approach that will enable reconstructing a healthy version of the totally unknown faulty tested network.

\section{Incipient fault characterization}

One of the main issues related to the detection of a soft fault in an NUT revolves around its nature (resistive, inductive, capacitive or conductive) and more importantly its variation relative to the cable parameters (RLCG parameters), i.e. its reflection coefficient. In fact, having in hand such pieces of valuable information could contribute to a great extent in avoiding drastic consequences attached to misinterpreted soft fault conditions. Particularly, the characterization of a soft fault is equally or even more important to the problem of detecting and locating the fault itself in a tested network.

On the other hand, cable aging is one of the recent topics that seems interesting in the domain of wire diagnostics [23], [24]. Aging as a term refers to the slow structural modification that can gradually decrease the efficiency of an element. Therefore, the characterization of a soft fault can permit estimating the electrical cable aging. Specifically, based on the cable (insulation and conductor) properties delivered by the manufacturer's data-sheets, the degradation process of a fault could be monitored based many factors such as: electric field, temperature, moisture, radiations, chemical corrosion, mechanical vibration, etc.. As a result, a timely maintenance 
anticipation for degraded faulty cable sections becomes possible as an early warning approach.

It is important to note that in a recent investigation of soft fault echoes associated with TDR measurements, the author in [25] has shown that weak TDR echoes do not necessarily correspond to weak soft faults. In other words, a superficial decay in a cable could produce the same TDR response compared to a critically damaged cable when tested at low frequencies. However, soft faults do generate echoes proportional to the bandwidth over which they are tested. Accordingly, in order bypass this analogy and enable a more precise evaluation of the fault's condition we will carry out our tests at sufficiently high frequencies ( $\mathrm{GHz}$ range). Besides, we aim at returning the fault's variation as a function of the cable's RLCG parameters rather than its severity or intensity. Notably, this is based on the fact that critical soft faults could return similar TDR echoes as those produced by weak soft faults.

To sum up, several challenges formed an impasse towards the proper application of soft fault diagnosis methods leading to ambiguous results, inaccuracy in location and failure in revealing the faults' characteristics in many cases. Under those circumstances, we will propose an efficient method for blindly testing a totally unknown wiring network to enable the characterization of soft faults embedded in it while properly detecting and accurately locating them by using a single testing port and without having to disconnect the NUT from its loads.

\section{METRICS OF THE PROPOSED APPROACH}

The proposed approach consists of two main phases. The first is dedicated to reconstruct the topology of the unknown tested network while the second is devoted to detect, locate and characterize the soft fault embedded in it. To do so, there is a need to efficiently solve the inverse problem of the measured network TDR response in order to retrieve the desired informations. Accordingly, this is possible by designing a network analysis direct numerical model.

\section{A. The Network Numerical Model}

The signal propagation in any network can be modeled by the RLCG circuit model of an infinitesimal section of the line. This model is based on Telegraph's equations that describe the evolution of voltage and current along the transmission line as function of the per unit length parameters demonstrated by the quantities $R$ (resistance), $L$ (inductance), $C$ (capacitance) and $G$ (conductance). To solve these equations we opted to adopt a joint finite difference time domain (FDTD)- ABCD matrix method developed as an in-house solver at CEA for wire network analysis. This same model has shown to be efficient in the investigations accomplished in [20]-[22] where the interested reader can refer to for more details. In fact, it allowed computing the TDR response of any desired NUT.

\section{B. The inverse problems}

The very first step commences by measuring the TDR response of the black-boxed NUT. Notably, the only piece of information needed is the type of cables constituting the tested network. Particularly, the cable's RLCG parameters permits encoding them in the aforementioned network model explained in sec. III-A so as to return a simulated TDR response similar to a measured one of the same tested network. On the other hand, a maximum number of extremities $M$ is important to reduce the computational complexity as will be shown later.

Having acquired the TDR response of the NUT which can be either collected by simulation or measured by a vector network analyzer (VNA), two inverse problems are to be solved on two different phases. The first phase is dedicated to reconstruct the topology of a healthy version of the tested unknown network by returning its structure, cable lengths and load impedances. Notably, this can be true as long as we are dealing with soft faults having weak TDR responses that usually have a minor contribution on the overall response of the network. The second phase is devoted for amplifying the signature of the soft fault by applying a post-processing approach. This approach is better known as "baselining" which takes the difference between the TDR response of the faulty NUT and that of a measured or simulated response of a healthy version of the same network. Fortunately, the first phase of the proposed approach permits having this simulated TDR healthy response. The aim afterwards is to extract the location and characteristics of the fault embedded in the network by solving a new inverse problem of the fault's baselined response. In what follows we will try to address all aspects of each phase.

\section{IMPLEMENTATION OF THE PROPOSED APPROACH}

The following section is meant to illustrate different steps of the proposed network-fault diagnosis method.

\section{A. First phase: Network reconstruction}

First of all, for the sake of clarification purposes, a numerical simulation based example will be provided. Accordingly, a single junction network composed of 3 ideal non-dispersive branches with a characteristic impedance $Z_{c}=50 \Omega$ is numerically implemented using a Matlab based transmission line simulation model. Two of the NUT's three extremities are connected to a $180 \Omega$ resistive load and a power transformer (open circuit) respectively. The third extremity is set as the testing port. Notably, the choice of the loads has no effect on the performance of the method as any other type of load can be chosen.

An inductive $L$ soft fault has been introduced on the first branch of the network with a $\Delta_{v}=30 \%$ and length $l_{f}=2 \mathrm{~cm}$. In fact, $\Delta_{v}$ is the fault's R,L,C, or G percentage variation relative to the RLCG parameters of the tested cable given as $\Delta_{v}=\left(X_{\text {actual }}-X_{\text {faulty }}\right) / X_{\text {actual }}$, with $X_{\text {actual }}$ being the actual $\mathrm{R}, \mathrm{L}, \mathrm{C}$, or $\mathrm{G}$ value of the cable while $X_{\text {faulty }}$ being the $R, L, C$, or $G$ value of the fault. A demonstration of the NUT showing the branch lengths and the fault properties is presented in Fig. 1. Practically, the NUT is unknown, i.e. it 


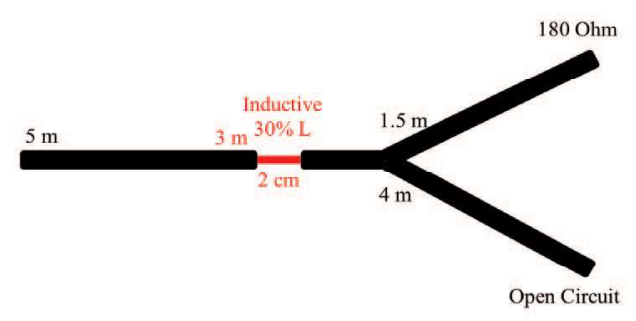

Fig. 1: A single junction network affected by a $2 \mathrm{~cm}$ soft inductive fault with a $\Delta_{v}=30 \%$ variation.

might be embedded in any system and would appear to us as a black-box having one free extremity which is the testing port.

The network reconstruction procedure of phase 1 will be accomplished based on previous work of methods presented in [20]-[22]. They integrate the graph theory and different optimization techniques (genetic algorithm (GA), particle swarm algorithm (PSO), etc.) to solve the inverse problem of the measured unknown network TDR response. This permits returning the most compelling NUT that resembles the tested network. Hereafter, we will briefly recall the methodology of such methods.

The TDR response of the NUT considered in Fig. 1 is computed using the FDTD-ABCD model of sec. III-A based on a Gaussian pulse having a bandwidth ranging from DC to $2 \mathrm{GHz}$. The corresponding reflectometry response $V_{\text {mes }}(t)$ is plotted in the reflectogram of Fig. 2.

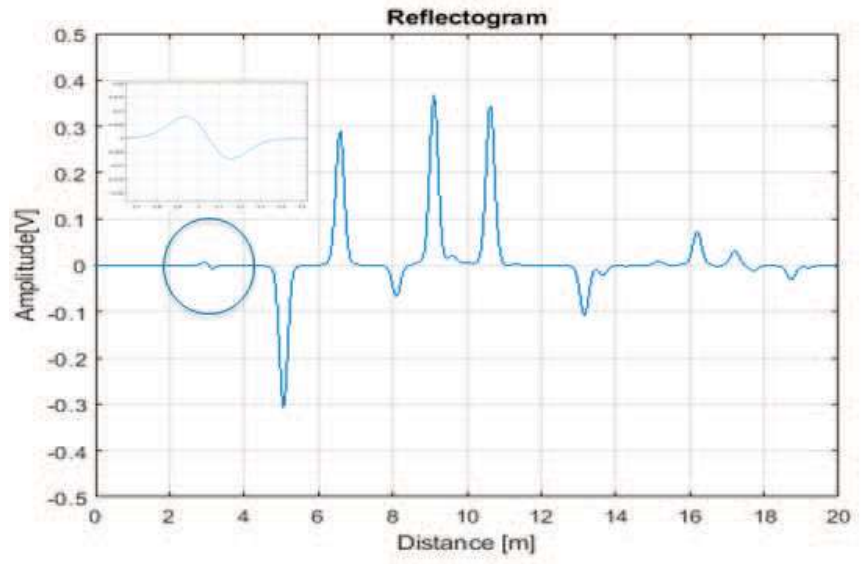

Fig. 2: A reflectogram showing the TDR response of the network of Fig. 1 with a zoomed plot of the fault's signature.

The whole idea starts from the fact that a wiring network is nothing else but a graph whose vertices are junctions and extremities, edges are branches whereas weights are their lengths. Consequently, based on the principles of graph theory, a generic network can be numerically modeled by three sets of variables (vertices, edges and weights) based on maximum number of network extremities $M$. Significantly, this is intended to limit the number of hypothesized networks and thus decrease the computational complexity. The TDR response of such a simulated NUT can be found based on the FDTD-
ABCD network model explained in sec. III-A. Accordingly, in order to construct the simulated network whose TDR response best matches the measured response of the unknown network, an inverse problem is to be solved.

Thus, the objective is to minimize the differences between the measured data set and that of the simulated one which is defined by an objective function and can be solved by an error-minimization algorithm. Indeed, global optimization algorithms as the GA or PSO provide a suitable tool for this purpose. The assigned objective function in our case study is given by eq. 1 where $V(t)_{\text {mes }}$ and $V(t)_{\text {sim }}$ are the time domain measured and candidate-simulated TDR responses. From now onwards, the temporal variable $t$ will be dropped for the sake of simplicity.

$$
F_{o b j}=\int_{t=0}^{\infty} \frac{\left\|V(t)_{s i m}-V(t)_{m e s}\right\|_{2}}{1+t^{2}} d t
$$

Since optimization techniques have some kind of stopping criteria, the desired value of the objective function will be assigned by taking into account the weak soft fault's response without having an impact on the network reconstruction process. On the other hand, the stopping criteria is determined by a stalking time during which no significant change in the population is noticed. As a result, a healthy numerical version of the tested unknown network would be retrieved with good estimates of the junction locations, branch lengths and load impedances.

The aforementioned algorithm is applied on the numerical example previously considered where the right network topology was retrieved with a length error not exceeding the $\mathrm{mm}$ range accompanied with good estimates of the load impedances (few ohm error). The results are demonstrated in the network of Fig. 3. More importantly, the reflectogram of Fig. 4 shows a close agreement between the reconstructed healthy network TDR response $V_{\text {sim }}^{H}$ and that of the faultynetwork $V_{m e s}$ with a single difference demonstrated by the absence of the fault's signature. The most compelling evidence on the efficiency of the proposed technique can be also deduced from the practical experimental validations presented in $[20]-[22]$.

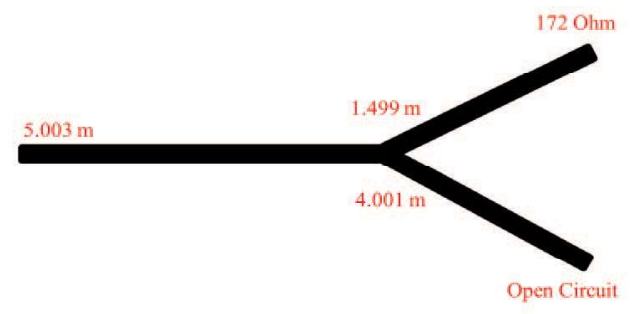

Fig. 3: The reconstructed healthy version of the faulty tested NUT of Fig. 1.

\section{B. Second phase: Fault characterization}

After having collected all information related to the tested unknown NUT, the remaining part is attached to retrieving 


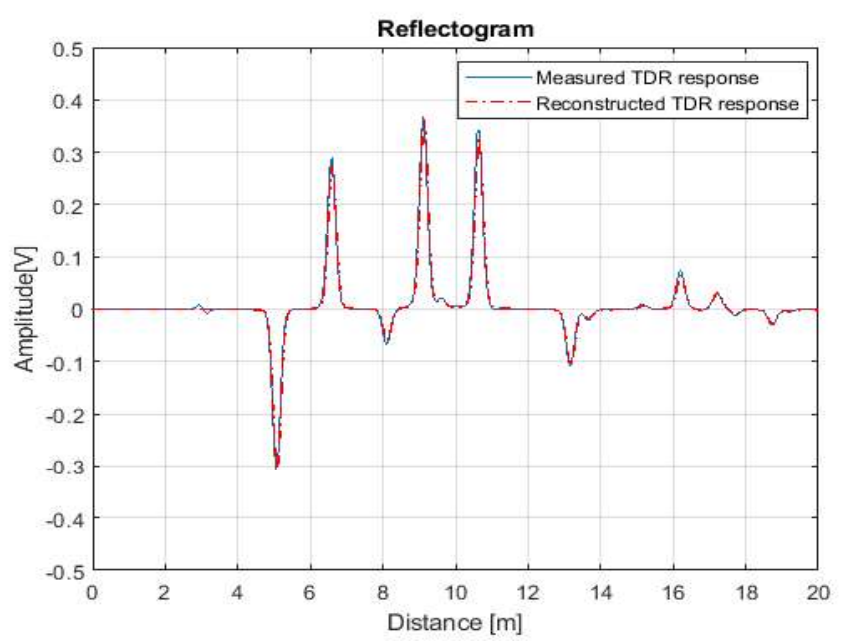

Fig. 4: The measured faulty and simulation-reconstructed healthy reflectometry responses.

the characteristics of the soft fault (nature, location, etc.) embedded in the network. This phase is composed of several steps.

1) Baselining and fault localization: As mentioned earlier, baselining, a contrast-enhancing technique, forms a suitable candidate for improving the detectability of weak signature soft faults. Originally it has been developed for radar applications in order to overcome the problem of clutter (unwanted signals) that can eventually mask the signature of a weak response target. In fact it is based on taking the difference between the system containing the target and clutter, and that of the system solely containing clutter [26]. And since a soft fault in a wire network is nothing else but a weak target in free space, and because impedance discontinuities can be regarded as clutter, baselining formed a natural fit to enhance the visibility of soft faults even in complex NUTs. Particularly, the proper application of baselining in guided wave propagation along transmission lines requires the availability of either a simulated or measured response of a healthy version of the network. Eventually, this is affordable thanks to the topology reconstruction algorithm presented in the sec. IV-A.

Accordingly, the baselined reflectometry response is computed as $V_{\text {Base }}=V_{\text {meas }}-V_{\text {sim }}^{H}$, where $V_{\text {meas }}$ contains the TDR response of the faulty unknown NUT, i.e. containing the signature of the soft fault embedded in the network, whereas the simulated TDR response $V_{s i m}^{H}$ forms the reference response containing the contribution of everything inside the reconstructed healthy NUT (impedance discontinuities if exist, junctions, etc.) except that of the fault. The baselined TDR response of the earlier considered NUT is presented in the reflectogram of Fig. 5. In fact, it can be noted that this procedure allowed removing most of the physiological echoes of the NUT that are not related to any fault (e.g., reflections at junctions and far ends of branches), which constitute the clutter response hindering the detection of eventual faults: as a result, minor echoes potentially related to faults are more

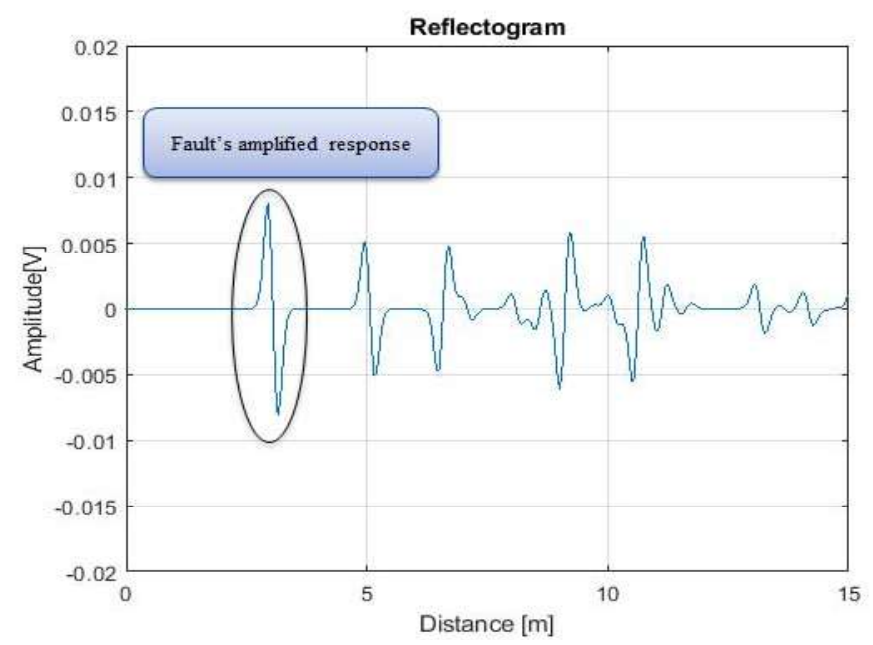

Fig. 5: Baselined TDR response of the NUT Fig. 1 showing an amplified signature of the soft fault.

clearly exposed, leading to an improved detection dynamic. Consequently, $V_{\text {Base }}$ ameliorated the signature of the soft fault which became more visible and be easily detected. Significantly, the plotted reflectogram $V_{\text {Base }}$ has also allowed an easy localization of the the soft fault which seems to be located at $3 \mathrm{~m}$ from the testing port.

2) Fault characterization: The following stage is dedicated to extract the soft fault's characteristics, namely nature (resistive, inductive, etc.) to later be able to monitor its effect on the system's performance and integrity. In order to do so, a new inverse problem is to be solved. We will consider the GA algorithm in our investigation due to its efficiency in terms of calculation time and simplicity.

The same graph theory-based algorithm of sec. IV-A is employed to construct a simulated wire network model resembling the reconstructed NUT (topology, branch lengths, and load impedances) but this time with a soft fault inserted on the revealed fault's position. The new NUT model is initialized with three new sets of variables. The first is that corresponding to the fault's nature $\{N\}=\{R, L, C, G\}$, the second is the length of the fault $\{D\}=\left\{L_{f}\right\}$ with $L_{f}$ being the length of the fault, while the third is the \% variation $\left\{\Delta_{v}\right\}$ with respect to the RLCG parameters of the tested cable. The corresponding simulated faulty network's TDR response $V_{s i m}^{f}$ is computed through the network analysis model of sec. III-A.

A GA optimization algorithm is then used to iteratively improve the simulated baselined reflectometry response $V_{\text {Base }}^{\text {sim }}=$ $V_{\text {sim }}^{f}-V_{\text {sim }}^{H}$ by changing the set of variables $\{N\},\{D\}$ and $\left\{\Delta_{v}\right\}$ in order to reduce the differences between the hypothesized faulty network model designated by $V_{\text {Base }}^{\text {sim }}$ and the baselined measured one demonstrated by $V_{\text {Base }}$. In other words, a similar objective function to that of eq. 1 is set between $V_{\text {Base }}^{\text {Sim }}$ and $V_{\text {Base }}$. The purpose of the optimization algorithm is to change the set of variables in order minimize the fitness value. Once the algorithm converges to a minimum that is to say $f v a l \approx 0$ we assume that we have reached the 
best candidates of the soft fault's characteristics.

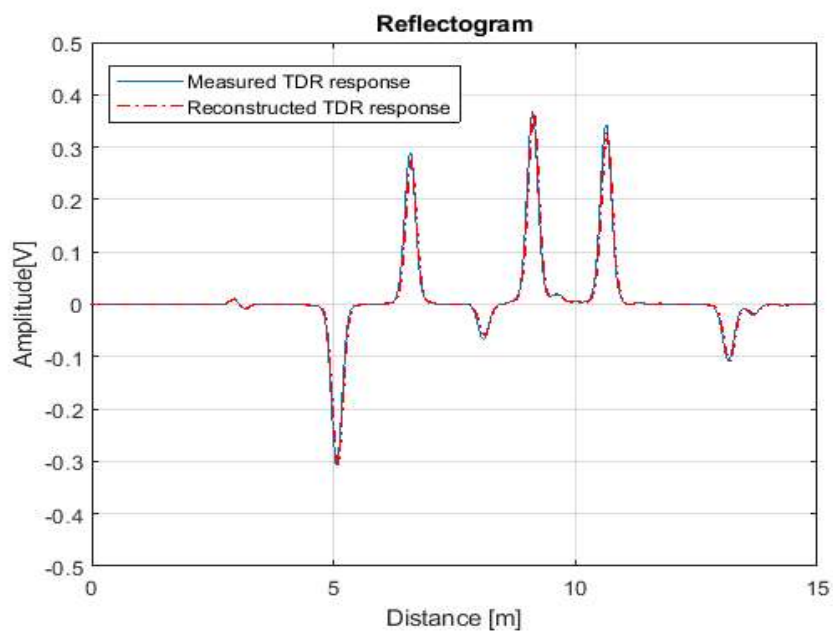

Fig. 6: The measured and simulation-reconstructed reflectometry responses of the final retrieved faulty network.

The application of the current procedure on the baselined response $V_{\text {Base }}$ of Fig. 5 leads to the identification of an inductive soft fault with a variation $\Delta_{v}=34 \%$ and a fault length $l_{f}=1.99 \mathrm{~cm}$. The reflectogram of Fig. 6 clearly demonstrates the fundamental alignment between the original measured TDR response and the final reconstructed one.

3) Network diagnosis: The final step is devoted to characterize the whole network by employing all the information obtained from the previous steps. Accordingly, a new network model is reconstructed by considering the retrieved topology, branch length and load impedances. The soft fault is then inserted on the estimated location with its retrieved characteristics. The network of Fig. 7 depicts the obtained results.

It is noteworthy that the proposed method is fully accomplished while the tested NUT is totally unknown during which its extremities are still connected to their loads. More importantly, we are able not only to blindly characterize the soft fault in the network but also reconstruct the NUT embedding it, with the lengths of the branches composing it and the load impedances connected to the NUT.

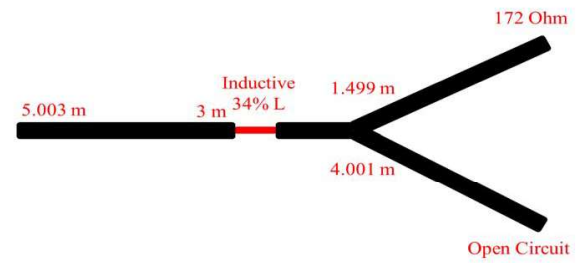

Fig. 7: The fully reconstructed network of the tested NUT of Fig. 1.

\section{RESULTS AND DISCUSSIONS}

In order to validate the potential applicability of the proposed approach in blindly diagnosing a faulty network, we opted to test it by experimental measurements.

\section{A. Experimental setup}

We conducted an experimental setup considering a three branch single-junction NUT in an effort to check the feasibility of the proposed technique on real-life networks. The network is composed of three RG-316 $50 \Omega$ coaxial cables connected to two loads of impedances $100 \Omega$ and $120 \Omega$ respectively. A soft fault was created by applying a crushing force on a $2 \mathrm{~cm}$ long section of the cable. The composed network is demonstrated in Fig. 8 and implemented as shown in Fig. 9. The free extremity of the NUT is connected as a testing port to a VNA Agilent Technologies E5071C. The acquisition of the TDR response is done by measuring the frequency domain S11 parameter. Applying an inverse fast Fourier transform (iFFT) to the measured data produces $V_{\text {mes }}$ which is plotted in the reflectogram of Fig. 11.

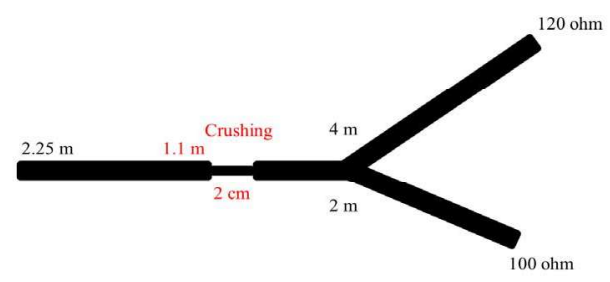

Fig. 8: The tested single junction faulty wire network.

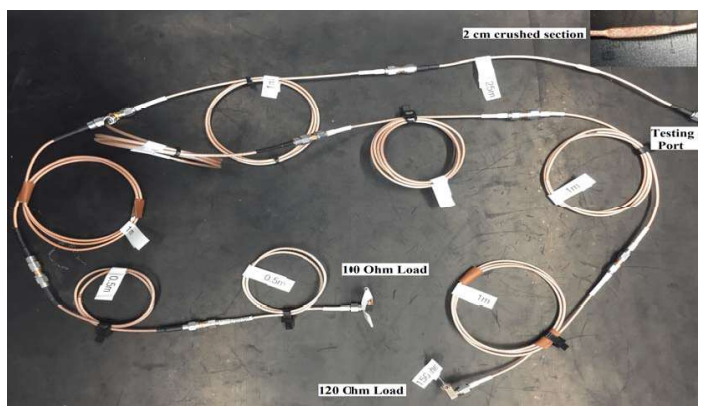

Fig. 9: Implementation of the NUT described in Fig. 1 with the faulty section shown on the upper right corner of the photo.

\section{B. Experimental validation}

After having measured the TDR response $V_{\text {mes }}$ of the tested NUT, phase 1 of the network-fault diagnosis process is applied. Significantly, the right network topology is reconstructed with very good estimates of the branch lengths and load impedances as demonstrated in Fig. 10.

As mentioned earlier, the objective of this step is to provide a healthy version of the network so as to enable computing a corresponding healthy simulated TDR response $V_{\text {sim }}^{H}$ using the FDTD-ABCD model. The reflectogram of Fig. 11 shows the agreement between the measured and reconstructed fault-free reflectometry responses.

Applying the difference system between $V_{\text {mes }}$ and $V_{\text {sim }}^{H}$ produces a fault enhancing baselined TDR response $V_{\text {Base }}$ as depicted in Fig. 12. Although several artifacts exist in the reflectogram but the location of the soft fault can be 


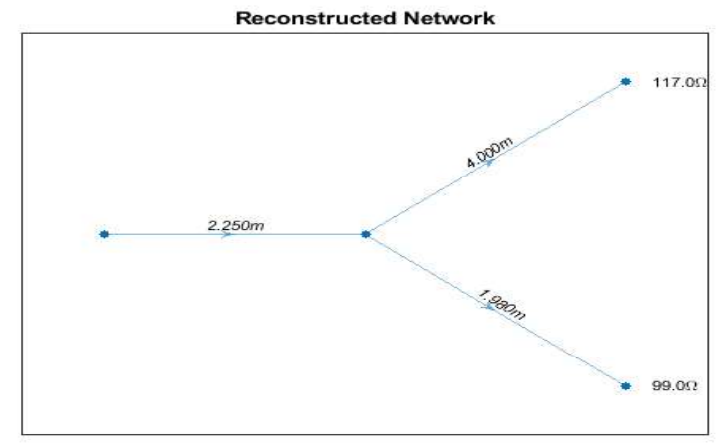

Fig. 10: The reconstructed healthy version of the faulty tested NUT of Fig. 8.

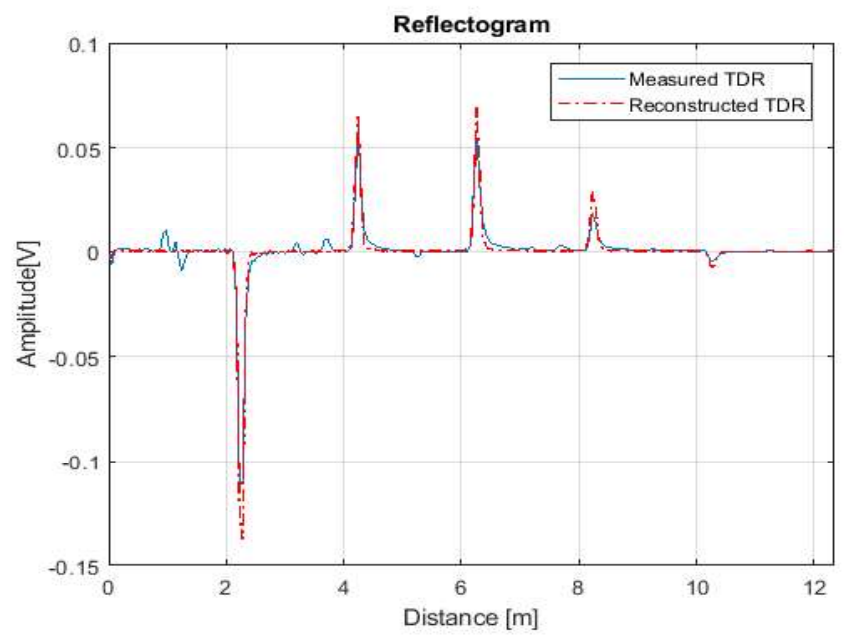

Fig. 11: The measured and simulation-reconstructed healthy reflectometry responses of the NUT implemented in Fig. 9.

easily extracted which is estimated to be at $1.104 \mathrm{~m}$ from the testing port, thanks to the signature improving potential of the proposed technique. In fact, these artifacts may be returned to the impossibility of obtaining a perfect resemblance between the reconstructed network and the tested one. Particularly, they are due to misalignments in the junction positions and differences in echo amplitudes of retrieved load impedances.

On the other hand, the fault characterization process is possible by solving the inverse problem of the baselined TDR response $V_{\text {Base }}$. Accordingly, applying the optimization-based algorithm detailed in sec. IV-B2 shows that the created fault is of inductive nature with a variation $\Delta_{v}=40 \%$, and a length $l_{f}=1.67 \mathrm{~cm}$. The close agreement between the measured and reconstructed TDR responses which is demonstrated in Fig. 13 validates the potential efficiency of the proposed approach in blindly reconstructing a faulty unknown network while returning valuable information (location and characteristics) about the fault embedded in it.

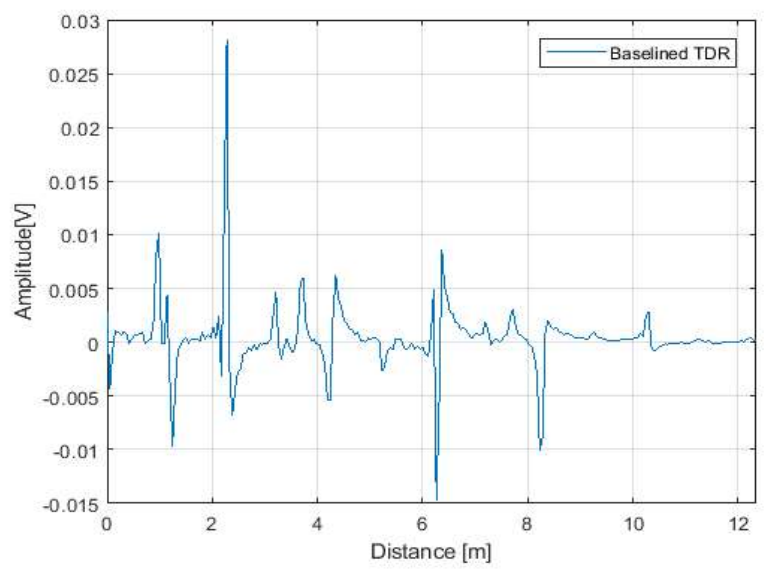

Fig. 12: Baselined TDR response of the NUT Fig. 8 showing an amplified signature of the soft fault.

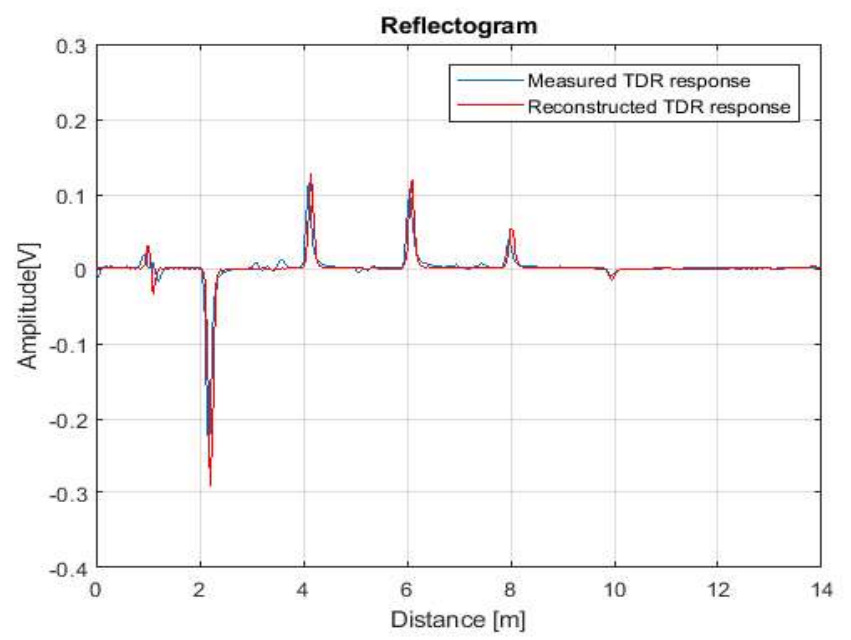

Fig. 13: The measured and final simulation-reconstructed reflectometry responses of the faulty network of Fig. 8.

\section{CONCLUSION}

In this paper we have proposed a technique mainly targeting the characterization of soft faults embedded in complex network configurations. In fact, this issue has become uprising amid the association of most fault diagnosis techniques to merely detection and location purposes.

The network-fault diagnosis method is an assembly of the well-known TDR techniques integrated with the graph theory and optimization based algorithms. Experimental results conducted on practical networks validate the potential practicability of the proposed method in efficiently characterizing (location, nature, severity) a soft fault embedded in a totally unknown network configuration. More importantly, this is accompanied by reconstructing the topology of the tested network while returning precise estimates of branch lengths and load impedances using a single testing port.

Future work will need to deal with several faults embedded in the same network and under unstable network circumstances 
manifested by the presence of vibrations, noise, etc.. Besides, executing the technique while the NUT is normally operating shall be very interesting.

\section{REFERENCES}

[1] F. Auzanneau, "Wire troubleshooting and diagnosis: Review and perspectives," Progress In Electromagnetics Research, vol. 49, pp. 253-279, 2013.

[2] M. Kafal, "Techniques d'imagerie pour la détection et la localisation de défauts non francs dans les réseaux de câblage," Ph.D. dissertation, Université Paris-Saclay, 2016.

[3] A. Cozza, and L. Pichon, "Echo response of faults in transmission lines: Models and limitations to fault detection," IEEE Transactions on Microwave Theory and Techniques vol. 64, no. 12, pp. 4155-4164, 2016.

[4] J. P. Steiner, "Time-Domain Reflectometry for Monitoring Cable Changes: Feasibility Study," Palo Alto, CA, USA: Electric Power Research Institute, 1990.

[5] N. G. Paulter, "An assessment on the accuracy of time-domain reflectometry for measuring the characteristic impedance of transmission lines," IEEE Transactions on Instrumentation and Measurement vol. 50, no. 5, pp. 1381-1388, 2001

[6] P. Smith, C. Furse, and J. Gunther, "Analysis of spread spectrum time domain reflectometry for wire fault location," IEEE sensors journal, vol. 5, no. 6, pp. 1469-1478, 2005.

[7] C. Furse, P. Smith, C. Lo, Y. C. Chung, P. Pendayala, and K. Nagoti, "Spread spectrum sensors for critical fault location on live wire networks," Structural Control and Health Monitoring: The Official Journal of the International Association for Structural Control and Monitoring and of the European Association for the Control of Structures, vol. 12 , no. 3-4, pp. 257-267, 2005.

[8] M. Franchet, N. Ravot and O. Picon, "The use of the Pseudo Wigner Ville Transform for detecting soft defects in electric cables," 2011 IEEE/ASME International Conference on Advanced Intelligent Mechatronics (AIM), Budapest, 2011, pp. 309-314.

[9] M. Franchet, N. Ravot and O. Picon, "Soft fault detection in cables using the cluster time-frequency domain reflectometry," IEEE Electromagnetic Compatibility Magazine, vol. 2, no. 1, pp. 54-69, 2013.

[10] S. Sallem and N. Ravot,"Self-adaptive correlation method for soft defect detection in cable by reflectometry," in IEEE SENSORS Conference, 2014, pp. 2114-2117.

[11] S. Sallem and N. Ravot,"Soft defects localization by signature magnification with selective windowing," in IEEE SENSORS Conference, 2015, pp.1-4.

[12] L. Abboud, A. Cozza, and L. Pichon, "A matched-pulse approach for soft-fault detection in complex wire networks," IEEE Transactions on Instrumentation and Measurement, vol. 61, no. 6, pp. 1719-1732, 2012.

[13] L. El Sahmarany, L. Berry, N. Ravot, F. Auzanneau, and P. Bonnet, "Time reversal for soft faults diagnosis in wire networks," Progress In Electromagnetics Research, vol. 31, pp. 45-58, 2013.

[14] R. Razzaghi, G. Lugrin, H. Manesh, C. Romero, M. Paolone, and F. Rachidi, "An efficient method based on the electromagnetic time reversal to locate faults in power networks," IEEE Transactions on Power Delivery, vol. 28, no. 3, pp. 1663-1673, 2013.

[15] M. Kafal, A. Cozza, and L. Pichon, "Locating faults with high resolution using single-frequency tr-music processing," IEEE Transactions on Instrumentation and Measurement, vol. 65, no. 10, pp. 2342-2348, 2016.

[16] M. Kafal, J. Benoit, A. Cozza, and L. Pichon, "A statistical study of dort method for locating soft faults in complex wire networks," IEEE Transactions on Magnetics, vol. 54, no. 3, pp. 1-4, 2018.

[17] L. A. Griffiths, R. Parakh, C. Furse, and B. Baker, "The invisible fray: A critical analysis of the use of reflectometry for fray location," Sensors Journal, IEEE, vol. 6, no. 3, pp. 697-706, 2006.

[18] M. Kafal, A. Cozza, and L. Pichon, "Locating multiple soft faults in wire networks using an alternative dort implementation," Instrumentation and Measurement, IEEE Transactions on, vol. 65, no. 2, pp. 399-406, 2016.

[19] M. Smail, H. Bouchekara, L. Pichon, H. Boudjefdjouf, and R. Mehasni, "Diagnosis of wiring networks using particle swarm optimization and genetic algorithms," Computers \& Electrical Engineering, vol. 40, no. 7, pp. 2236-2245, 2014.

[20] M. Kafal, J. Benoit, G. Beck, and A. Dupret, "Constructing the topology of complex wire networks using reflectometry response and newtonbased optimization algorithms," in AUTOTESTCON, 2017 IEEE. IEEE, 2017 , pp. $1-6$.
[21] M. Kafal, J. Benoit, and C. Layer, "A joint reflectometry-optimization algorithm for mapping the topology of an unknown wire network," in SENSORS, 2017 IEEE. IEEE, 2017, pp. 1-3.

[22] M. Kafal, J. Benoit, G. Beck, and E. Cabanillas, "Blind diagnosis of a black-boxed fully-loaded wiring network for configuration structuring and fault monitoring," in 2018 IEEE International Instrumentation and Measurement Technology Conference (I2MTC).

[23] R.F. Gazdzinski, "Cable and method of monitoring cable aging," U.S. Patent, no. 5,902,962. 11 May 1999

[24] L. El Sahmarany, F. Auzanneau, and P. Bonnet,, "Novel reflectometry method based on time reversal for cable aging characterization," in 2012 IEEE 58th Holm Conference on Electrical Contacts (Holm), pp. 1-6.

[25] A. Cozza, "Never Trust a Cable Bearing Echoes: Understanding Ambiguities in Time-Domain Reflectometry Applied to Soft Faults in Cables," IEEE Transactions on Electromagnetic Compatibility, 2018.

[26] M. Kafal and J. Benoit, "Baselining: A critical approach used for soft fault detection in wire networks," EEETEM, 2017. 